\title{
The effect of lameness prevalence on technical efficiency at the dairy farm level: An adjusted data envelopment analysis approach
}

\author{
A. P. Barnes, ${ }^{\star 1}$ K. M. D. Rutherford, $†$ F. M. Langford, $†$ and M. J. Haskell $†$ \\ *Land Economy and Environment Group, Scottish Agricultural College, Edinburgh, EH9 3JG, United Kingdom \\ †Sustainable Livestock Systems, Scottish Agricultural College, Edinburgh, EH9 3JG, United Kingdom
}

\begin{abstract}
A key indicator of resource use within farming is technical efficiency, which measures the amount of physical output attainable from a given set of inputs. The social aspects, in particular the treatment of animals, have generally been ignored within these measurement schemas. In addition, animal welfare will affect the production technology under which farms operate, and some allowance for this is needed within the measurement approach. This is the first paper to apply animal welfare as a discriminating technology within a technical efficiency framework. Using results from an animal welfare monitoring study coupled with resource usage data, it presents an adjusted measure of technical efficiency applied to a sample of British dairy farms and compares differences in lameness management strategies for herds. We employ both a categorical and nondiscretionary variant of the data envelopment analysis approach to measure technical efficiencies and adjust for various degrees of lameness prevalence among these farms. This paper finds that farms with low rates of lameness (below 10\% of the cattle herd) tend to have significantly higher technical efficiencies than those with lameness rates of above $10 \%$ of the herd. Farms that have levels of lameness of between 10 to $20 \%$ of the herd and higher levels of lameness (above 20\% of the herd) did not differ significantly. Furthermore, low lameness farms are inefficient in terms of labor and stocking density, but this is outweighed by the gain in milk yield obtained on these farms. Consequently, we argue for a whole-farm, rather than a partial indicator, approach to assessing efficiency when noneconomic factors such as lameness are accounted for. From a policy perspective, we support programs that encourage active lameness management.
\end{abstract}

Key words: categorical approach, nondiscretionary approach, data envelopment analysis, lameness prevalence

Received February 11, 2011.

Accepted August 5, 2011.

${ }^{1}$ Corresponding author: Andrew.Barnes@sac.ac.uk

\section{INTRODUCTION}

The role of welfare indicators and economic performance has been explored in several studies, mostly finding a positive relationship between the well-being of the cow and economic performance (Hansson, 2007; Hansson and Ohlmer, 2008). However, an indicator of welfare that has generated mixed results is lameness in cattle. Lameness is a well-recognized problem within the dairy industry and its incidence can be reduced through active management (Archer et al., 2010). Lameness can be defined as a gait abnormality in the cow and is widely recognized by farmers and other experts within the dairy industry as being an important contributor to poor dairy cow welfare by being associated with pain, poor body condition, and reduced fertility (Whay et al., 2003; FAWC, 2009; Leach et al., 2010a,b). Studies exploring the implications of lameness within dairy cattle have found a mixture of positive and negative associations between milk yield and incidence of lameness (Enting et al., 1997; Rajala-Schultz et al., 1999; Warnick et al., 2001; Green et al., 2002; Hernandez et al., 2002; Green et al., 2010).

Very few studies have approached animal welfare as a distinct influence on resource use at the farm level and most have ignored this as an inefficiency effect. Notable exceptions are Lawson et al. (2004) and Hansson et al. (2010), who apply the basic assumption that all farmers face the same technological combinations of inputs and outputs. However, this creates bias in the technical efficiency measure, because lameness prevalence affects these production relationships. Accordingly, this is the first paper to explore the issue of animal welfare as a discriminating technology within a technical efficiency measurement framework. This is important, because accurate measurement of these effects will provide information at both the policy and the farmer advisory level.

The aim of this paper was to extend the technical efficiency approach to include a measure of welfare in a study of 80 dairy farms across Great Britain, using observed data on inputs and outputs and collected lameness scores. We explore the question of whether 
maintaining low lameness prevalence on an individual farm leads to higher levels of efficiency and how this could influence policy to encourage better management. Hence, this study provides a measure that integrates societal needs, for better animal welfare, within a framework that considers resource usage across farms.

\section{MATERIALS AND METHODS}

The background to measuring efficiency is based on the work of Farrell (1957). A frontier can be drawn from observed data on farms operating at their optimal levels of efficiency. This frontier represents the best practice technology of that particular industry at a particular point in time. Hence, farms that are on the frontier present the most achievable levels of efficient resource use for that industry. It also provides an upper limit for farms that operate below that frontier. For these farms to reach the frontier usually requires some combination of management and technological progress to be achieved at the farm level. Farrell's approach can be applied within a farm benchmarking context, and farm technical efficiency can be ranked relative to that frontier within a unified measure that runs from 0 to 1 , where 1 is the greatest efficiency attainable within a particular sector at a particular point in time.

Farrell's approach can be applied using parametric, semiparametric, or nonparametric approaches to constructing a best practice frontier. The main advantage of the parametric approach is that a stochastic element is removed from the efficiency score to account for variations in such factors as weather and disease. However, the stochastic production frontier technique makes assumptions regarding the adoption of an appropriate functional form and the distributions of the efficiency disturbance term, which may introduce bias to the evaluation (Coelli et al., 1998; Kuosmanen and Kortelainen, 2011).

An alternative is the nonparametric data envelopment analysis (DEA) approach. The advantage of the DEA technique is that, although it has no stochastic element to account for disease and weather, no assumptions are made of the underlying technology function. Several studies have explored dairy systems using the DEA approach (Bravo-Ureta and Rieger, 1991; Cloutier and Rowley, 1993; Hallam and Machado, 1996; Fraser and Cordina, 1999; Jaforullah and Whiteman, 1999; Cuesta, 2000; Barnes et al., 2006; Barnes, 2008; Hansson et al., 2010).

More critically, the desirable aspect of this approach is its "unit-invariance"; that is, variables of separate units can be included in the assessment of efficiency. This aspect has led the majority of studies aimed at adjusting technical efficiency measures for noneconomic factors to apply the DEA approach (Piot-Lepetit et al., 1997; Reinhard et al., 2000; De Koeijer et al., 2003). However, all these studies have focused on adjusting technical efficiency for environmental harm and tended to ignore aspects of animal health and welfare. Second, DEA can accommodate categorical variables that represent different states of production (Banker and Moray, 1986). Thus, various states of lameness can be included in the DEA evaluation to adjust the measures of efficiency found between farms. This method is discussed below.

Data envelopment analysis (Charnes et al., 1978) is a method that allows multi-input and multi-output production systems to be benchmarked, by constructing a piecewise linear surface over observed data points. This frontier surface is assembled by solving a sequence of linear programming problems, one for each firm or decision-making unit (DMU), in the sample. The linear program calculating the efficiency score of the $i$ th DMU in a sample of $N$ DMUs can be specified as follows:

$$
\begin{gathered}
\text { Min } \theta \\
\text { Subject to } \sum_{n=1}^{N} \lambda_{n} y_{q n} \geq y_{q 0}, q=1, \ldots, Q, \\
\sum_{n=1}^{N} \lambda_{n} x_{p n} \leq \theta x_{p 0}, \quad p=1, \ldots, P,
\end{gathered}
$$

where $\lambda_{n}, \mathrm{x}_{p n}, \mathrm{y}_{p n} \geq 0, n=1, \ldots, N ; \theta$ is a scalar and $\boldsymbol{\lambda}$ represents an $N \times 1$ vector of weights; $\mathbf{x}$ and $\mathbf{y}$ are matrices of input and output quantities for all farms respectively; $\mathbf{x}_{n}$ is a vector of input quantities for the $n$th farm, and $\mathbf{y}_{n}$ is a vector of output quantities for the $n$th farm. The above problem is solved using a linear programming approach for each farm, and a technical efficiency score is measured as the radial distance between that farm and the frontier. The scores range from 0 to 1 , with 1 indicating that the unit is operating on the frontier. It should also be noted that this is the constant returns to scale (CRS) specification. However, this is only appropriate if we assume that all farms are operating at their optimal scale; that is, they do not need to change size to realize efficiencies. The variable returns to scale (VRS) model employs a convexity constraint; namely,

$$
\sum_{n=1}^{N} \boldsymbol{\lambda}_{n}=1,
$$

where $\boldsymbol{\lambda}_{n}$ is a row vector of $1 \mathrm{~s}$. The main advantage of the VRS model is that scale-inefficient farms are only compared with efficient farms of a similar size. Hence, 
some adjustment is made for scale when calculating efficiency rankings. Most studies tend to calculate both CRS and VRS models, which allows researchers to decompose the results into "pure" technical efficiency and scale efficiency. This can be done by taking the ratio of CRS to VRS output. Hence, this gives some indication of the gains to be achieved from either increasing or decreasing in scale. This paper presents all 3 outputs. More discussion of DEA can be found in Coelli et al. (1998) and Cooper et al. (2000).

The standard DEA model assumes that all inputs (outputs) for inefficient farms can be minimized (maximized) radially to shift that farm into the efficiency frontier. This assumes that all farms have the management discretion to reduce these inputs by appropriate quantities to reach the frontier. For a standard input-minimizing approach then, key inputs may be reduced at farm level; in some cases, input reduction is actively encouraged by policy makers (e.g., nitrogen). Banker and Moray (1986) were the first to examine nondiscretionary, or environmental, variables that offer a mixture of constraints under which producers could operate. Within the context of this paper, a comparison can be made when treating lameness as something that can be controlled (discretionary) or is noncontrollable (nondiscretionary) by the farmer. This is useful because it allows us to compare differences in efficiency between active farmer management and no intervention for lameness within a dairy herd. Continuous nondiscretionary variables can be included within the DEA by introducing a further constraint, $\mathrm{z}$ :

$$
\sum_{n=1}^{N} \lambda_{n} z_{r n} \leq z_{r 0}, r=1, \ldots, R
$$

where $\mathbf{Z n}$ is a vector of nondiscretionary inputs for the $n$th farm. This replicates the constraints above but allows only weighted averages of efficient observations to enter the referent point (Lober and Staat, 2010).
A second approach is to apply the VRS model and partition the data along different lameness classes; that is, representing a group of producers operating under similar management conditions (Banker and Moray, 1986). The advantage of this approach is that it addresses the issue of describing separate frontiers to accommodate different production possibilities. Both these methods are applied and described below.

\section{Data Collection and Analysis}

Data were collected from 80 dairy farms within Britain (for full details of farm recruitment and characteristics see Rutherford et al., 2008). All of the farms were visited once during the autumn (September to October) and once during the spring (March to June). All farms grazed the cows at pasture during the summer and housed them during the winter. Over $95 \%$ of these cows were Holsteins. On each farm, lameness prevalence was assessed using locomotion scoring by trained observers. Cow locomotion was scored on a 4-point scale (Table 1), which was a simplification of a previously used scale (Manson and Leaver, 1988). All cows in the milking herd were scored as they left the milking parlor at either the morning or afternoon milking.

The mean interobserver reliability between 2 observers at the end of the training periods was $67.2 \%$ when all 4 lameness score categories were analyzed and $90.5 \%$ when scores 1 and 2 , and 3 and 4 were grouped into 2 categories of "sound" and "lame," respectively (Rutherford et al., 2009). A total locomotion score for each farm was calculated by taking the percentage of cows with locomotion scores of 3 and 4 (lame) to the total herd. Over all farm visits, lameness prevalence varied between farms from 1.4 to $48.6 \%$.

An average of the 2 sets of scores were taken and then classified into 3 types; namely, low, medium, and high lameness (Table 2). Essentially, these categories developed from partitioning the data across various ranges. However, the overall range is similar to that

Table 1. Description of locomotion score categories

\begin{tabular}{|c|c|}
\hline Category & Description \\
\hline 2: Mild deviation from normal walking & $\begin{array}{l}\text { Cow walks with a small gait abnormality. Gait pattern is almost normal } \\
\text { but cow shows slight but noticeable deviations from normal tracking } \\
\text { or clear adduction or abduction, with some limb rotation. }\end{array}$ \\
\hline 3: Gait abnormality & $\begin{array}{l}\text { Cow is clearly walking abnormally, with clear deviations from normal } \\
\text { tracking and abduction or adduction (including limb rotation). Stride } \\
\text { length can be reduced and stride rhythm may be irregular. }\end{array}$ \\
\hline
\end{tabular}

\footnotetext{
${ }^{1}$ Tracking $=$ how close the hind foot is placed in the print of the fore foot. Normally the hind foot is placed exactly in the print of the fore foot. Abduction $=$ a tendency to move the foot lateral from the line of forward movement. Adduction $=\mathrm{a}$ tendency to move the foot medial to the line of forward movement.
} 
Table 2. Categories of lameness prevalence according to locomotion score

\begin{tabular}{ll}
\hline Lameness & Description \\
\hline High & A locomotion score of 3 or 4 for more than $20 \%$ of the herd \\
Medium & A locomotion score of 3 or 4 for 10 to $20 \%$ of the herd \\
Low & A locomotion score of 3 or 4 for less than $10 \%$ of the herd \\
\hline
\end{tabular}

found by Whay et al. (2003) and in that study, 90\% of cattle welfare experts believed that some form of intervention should be undertaken at a farm lameness prevalence of $19.5 \%$ or above.

The box plot in Figure 1 shows the median and interquartile ranges of the lameness scores for the 3 categories, indicating low, medium, and high levels of lameness. Low lameness status has a median of only 0.06 (6\% of the herd), whereas medium status had a median of $0.16(16 \%)$, and farms in high lameness had a median of 0.26 ( $26 \%$ of the herd with scores of 3 and 4).

As part of the farm visit, information on farm characteristics, feeding, and management procedures were obtained by interviewing the farmer (Langford et al., 2009). Data on resource usage over an annual period were also collected from these sites, and key input and output variables were collated to provide the basis for the data envelopment analysis. These data are summarized in Table 3 and have been subdivided by lameness prevalence category.

A definite difference was observed in the characteristics of each category of farm in terms of average size of operation. The farms with low lameness status tended to have a lower average number of cows within the herd compared with higher lameness categories. These farms tend to manage a larger herd and produce a higher yield per cow, which follows the argument of Fourichon et al. (2000) that farms with above-average risk for lameness disorders are those with high milk production per cow. However, input use per cow is relatively similar across the 3 lameness states. Although these figures are presented at the mean for descriptive purposes, the advantage of the DEA approach is the ability to simultaneously consider input and output combinations of individual farms within a single optimization problem. Thus, DEA identifies the most efficient performers at an individual farm rather than at the mean, partial indicator, level.

The process of evaluation followed 2 main strategies. The totals of the 80 farms were first measured within a single DEA, which was input-oriented; that is, the optimization problem was to minimize inputs subject to a fixed level of output. Thus, all farms were assumed to face a similar set of technological possibilities and management for lameness is controllable; that is, lameness prevalence could be actively decreased. In this case, all farms faced a similar set of technological possibilities and hence were compared against a common frontier regardless of lameness levels. This could be used as a baseline measure of performance across the 80 farms. Then, subsets of DEA were run with each lameness category, which provides the basis for the discriminatory technology. Hence, the frontier was constructed of farms from only within the same lameness category, representing a management constraint. Reflecting the argument of Banker and Moray (1986) that categories must reflect the same or lower levels of quality, the 3 DEA covered (1) the low lameness set, (2) the medium and low lameness set, and (3) the high lameness set.

Second, the percentage of cows with lameness scores of 3 or 4 to the total herd was used as a continuous input alongside the standard economic inputs outlined in Table 2. The advantage of this approach was that it gave an indication of how much lameness should decrease per farm to attain higher levels of technical ef-

Table 3. Descriptive statistics (SD in parentheses) for outputs and inputs for dairy farms, categorized by mean values for lameness prevalence (Table 2)

\begin{tabular}{|c|c|c|c|c|c|c|c|}
\hline Status & $\begin{array}{c}\text { Yield } \\
\text { (L/cow) }\end{array}$ & $\begin{array}{c}\text { Grazing } \\
\text { area (ha) }\end{array}$ & $\begin{array}{c}\text { Annual } \\
\text { feed and } \\
\text { forage }(t)\end{array}$ & $\begin{array}{l}\text { Annual } \\
\text { labor (h) }\end{array}$ & $\begin{array}{l}\text { Annual } \\
\text { replacement } \\
\text { cows (no.) }\end{array}$ & $\begin{array}{l}\text { Average } \\
\text { lameness } \\
\text { score }(\%)\end{array}$ & $\begin{array}{l}\text { Herd size } \\
\text { (no.) }\end{array}$ \\
\hline All & $\begin{array}{c}7,513 \\
(1,153)\end{array}$ & $\begin{array}{l}100 \\
(46)\end{array}$ & $\begin{array}{c}2,063 \\
(1,107)\end{array}$ & $\begin{array}{c}8,667 \\
(3,794)\end{array}$ & $\begin{array}{c}38 \\
(19)\end{array}$ & $\begin{array}{l}16.1 \\
(9.1)\end{array}$ & $\begin{array}{l}175 \\
(77)\end{array}$ \\
\hline Low & $\begin{array}{c}7,173 \\
(1,019)\end{array}$ & $\begin{array}{c}80 \\
(39)\end{array}$ & $\begin{array}{c}1,464 \\
(1,003)\end{array}$ & $\begin{array}{c}6,784 \\
(2,525)\end{array}$ & $\begin{array}{c}27 \\
(12)\end{array}$ & $\begin{array}{c}5.8 \\
(2.3)\end{array}$ & $\begin{array}{l}137 \\
(51)\end{array}$ \\
\hline Medium & $\begin{array}{c}7,518 \\
(1,228)\end{array}$ & $\begin{array}{l}106 \\
(50)\end{array}$ & $\begin{array}{c}2,228 \\
(1,085)\end{array}$ & $\begin{array}{c}9,371 \\
(4,346)\end{array}$ & $\begin{array}{c}42 \\
(20)\end{array}$ & $\begin{array}{l}15.4 \\
(2.8)\end{array}$ & $\begin{array}{l}189 \\
(88)\end{array}$ \\
\hline
\end{tabular}




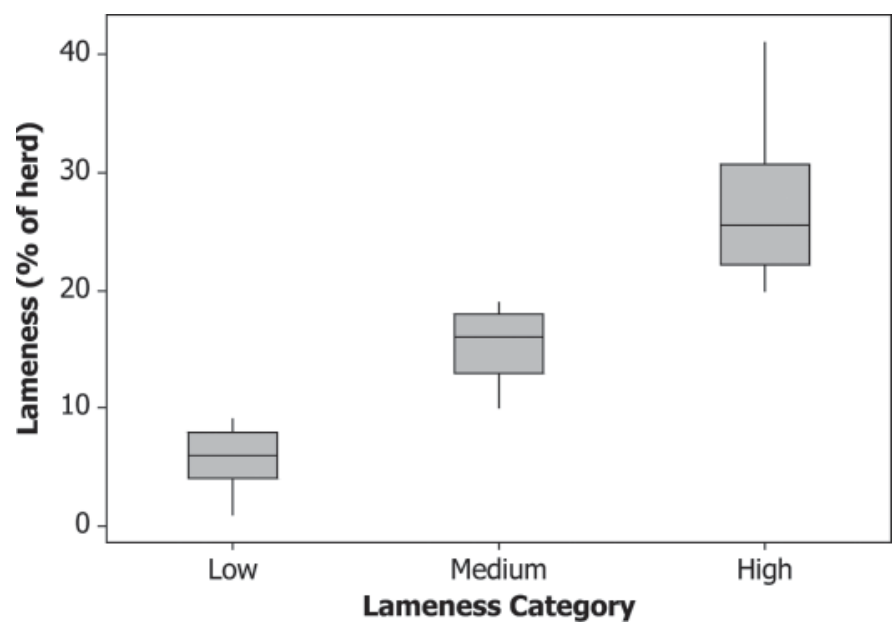

Figure 1. Lameness category by animal welfare category for sample of 80 British dairy farms, showing box plot of median and interquartile range.

ficiency. However, it may not be a realistic assumption to expect lameness to decrease, so the second DEA run holds the lameness score as noncontrollable (nondiscretionary) and then evaluates performance. Hence, under this approach, lameness is treated as a variable under which no producer has control.

An issue with the application of DEA is that scores may bias upward, especially when samples are small and variable numbers are large (Tauer and Hanchar, 1995). Simar and Wilson (1998) proposed the "smoothed bootstrap approach" to identify the statistical properties of the efficiency scores and to correct for bias in the sample, which may occur when applying a deterministic approach to agricultural data. This is especially useful in this context because the partitioning of data along lameness categories leads to smaller sample sizes. Hence, the scores reported below are those corrected for this bias after applying smoothed bootstrapping, with 2,000 replications, along with their associated upper and lower confidence limits, again derived from the Simar and Wilson (1998) methodology.

\section{RESULTS}

The categorical DEA approach applied here constructs 3 frontiers and compares the performance of farms purely within their different lameness classes. Table 4 shows that farms with a low lameness score generate a mean technical efficiency score of 0.93 under the VRS model, whereas those with higher levels of lameness have scores of around 0.80 .

A one-way ANOVA test on the differences in efficiency scores from low lameness farms $(<10 \%$ of the herd) compared with those with higher lameness scores gave an observed significance of $P=0.001$. Conversely, a one-way ANOVA test between the medium (10 to $<20 \%$ of the herd with severe lameness) and higher lameness categories (at least 20\% of the herd with severe lameness) gave an observed significance of $P=$ 0.399 .

Table 5 shows the results of using the percentage of lameness in each herd as a continuous variable, either using it as a variable that could be managed (discretionary) or as one that is outside the boundaries of management (nondiscretionary). These results were then tabulated against the high, medium, and low lameness categories outlined above. In general, the farms in the sample achieved a mean technical efficiency score of 0.79 , compared with 0.82 when lameness was handled as an uncontrollable input within the DEA.

Regardless of the approach taken, farms with lower levels of lameness tend to generate higher levels of technical efficiency. Farms in the low lameness category have a higher mean efficiency (0.905), compared with the medium lameness category (0.79) and the high lameness category (0.77). Table 6 shows the results of one-way ANOVA tests on these results.

The main finding seems to be that farms with a lameness prevalence $<10 \%$ of the herd generate higher levels of technical efficiency. This provides some indication that a positive relationship exists between improving managing lameness and resource usage. The evidence for those with a prevalence of lameness $>10 \%$ of the herd is somewhat mixed and depends on how lameness is evaluated. Consequently, from a farm management perspective, farmers should endeavor to reduce lameness to below the $10 \%$ level to benefit from higher rates of resource usage.

All inefficient farms can, in theory, be projected onto the frontier by reducing levels of input to maintain output. The mean level of reduction needed in inputs is presented below for all farms and by lameness state, using the discretionary approach that includes lameness as a continuous variable (Figure 2).

Table 4. Summary statistics for data envelopment analysis using a categorical approach, biased corrected technical efficiency (TE) estimates, standard deviations (in parentheses), and 95\% confidence intervals

\begin{tabular}{|c|c|c|c|}
\hline \multirow[b]{2}{*}{ Lameness } & \multirow{2}{*}{$\begin{array}{c}\text { Corrected } \\
\text { TE }\end{array}$} & \multicolumn{2}{|c|}{$95 \%$ CI } \\
\hline & & Lower & Upper \\
\hline Low & $\begin{array}{c}0.926 \\
(0.098)\end{array}$ & 0.859 & 0.957 \\
\hline Medium & $\begin{array}{c}0.812 \\
(0.127)\end{array}$ & 0.711 & 0.809 \\
\hline High & $\begin{array}{c}0.783 \\
(0.133)\end{array}$ & 0.663 & 0.778 \\
\hline
\end{tabular}


Table 5. Summary statistics for discretionary and nondiscretionary approach to lameness score (low, medium, or high lameness prevalence; Table 2), variable returns to scale, biased corrected efficiency estimates, standard deviations (in parentheses) and 95\% confidence intervals

\begin{tabular}{|c|c|c|c|}
\hline \multirow[b]{2}{*}{ Analysis } & \multirow{2}{*}{$\begin{array}{c}\text { Corrected } \\
\text { TE }\end{array}$} & \multicolumn{2}{|c|}{$95 \%$ CI } \\
\hline & & Lower & Upper \\
\hline \multicolumn{4}{|c|}{ Discretionary } \\
\hline Low & $\begin{array}{c}0.935 \\
(0.092)\end{array}$ & 0.887 & 0.986 \\
\hline Medium & $\begin{array}{c}0.785 \\
(0.129)\end{array}$ & 0.754 & 0.838 \\
\hline High & $\begin{array}{c}0.766 \\
(0.124)\end{array}$ & 0.738 & 0.793 \\
\hline \multicolumn{4}{|c|}{ Nondiscretionary } \\
\hline Low & $\begin{array}{c}0.875 \\
(0.130)\end{array}$ & 0.855 & 0.942 \\
\hline Medium & $\begin{array}{c}0.785 \\
(0.132)\end{array}$ & 0.739 & 0.815 \\
\hline High & $\begin{array}{c}0.765 \\
(0.123)\end{array}$ & 0.737 & 0.788 \\
\hline
\end{tabular}

Farms with low lameness tended to have higher levels of technical efficiency and were, therefore, closer to the frontier. However, what is noticeable is that the levels of reduction needed for these farmers are higher for grazing area and labor in these farms (Figure 2). A clear relationship exists between lower levels of lameness and stocking density and labor usage on these farms. Higher levels of stocking density would lead to a more efficient use of resources, but this will have an effect on lameness. Similarly, the high levels of labor time spent per cow on farms with low levels of lameness also indicate an inefficient use of the resources within the standard schema of technical efficiency measurement. However, the gains from yield per unit of input at a whole-farm
Table 6. Results of one-way ANOVA on technical efficiency scores from discretionary (DS) and nondiscretionary (NDS) analysis

\begin{tabular}{lcc}
\hline Analysis $^{1}$ & $F$-value & $P$-value \\
\hline NDS (L, M, H) & 12.65 & 0.000 \\
NDS (M, H) & 0.19 & 0.667 \\
DS (L, M, H) & 14.93 & 0.000 \\
DS (M, H) & 0.002 & 0.950 \\
NDS (H) DS (H) & 0.001 & 0.982 \\
NDS (M) DS (M) & 0.34 & 0.562 \\
NDS (L) DS (L) & 0.48 & 0.745
\end{tabular}

$\overline{{ }^{1} \mathrm{~L}=\text { low }(<10 \% \text { herd with severe lameness prevalence); } \mathrm{M}=\text { medium }}$ (10 to $<20 \%$ herd with severe lameness prevalence); $\mathrm{H}=$ high $(>20 \%$ herd with severe lameness prevalence).

level are clearly still higher for these farms as overall technical efficiency is greater than farms with higher lameness prevalence.

What is notable about the farms with high lameness is the high level of reduction needed in feed and forage (Figure 2). Dairy farms with higher levels of lameness tend to have higher yields and therefore require more concentrates to support that greater yield. However, we have shown that taking a relative efficiency approach including lameness as a factor in production, these gains in yield do not improve overall technical efficiencies.

Under the discretionary approach, lameness is treated as an input that could be minimized. This is presented as the percentage reduction in lameness required to reach higher levels of technical efficiency and, as expected, farms with high lameness need to reduce lameness by a greater amount than farms in other categories. This would require an expansion in seemingly nonefficient input usage; namely, reduced stocking densities and more labor time allocated to monitor cows for early

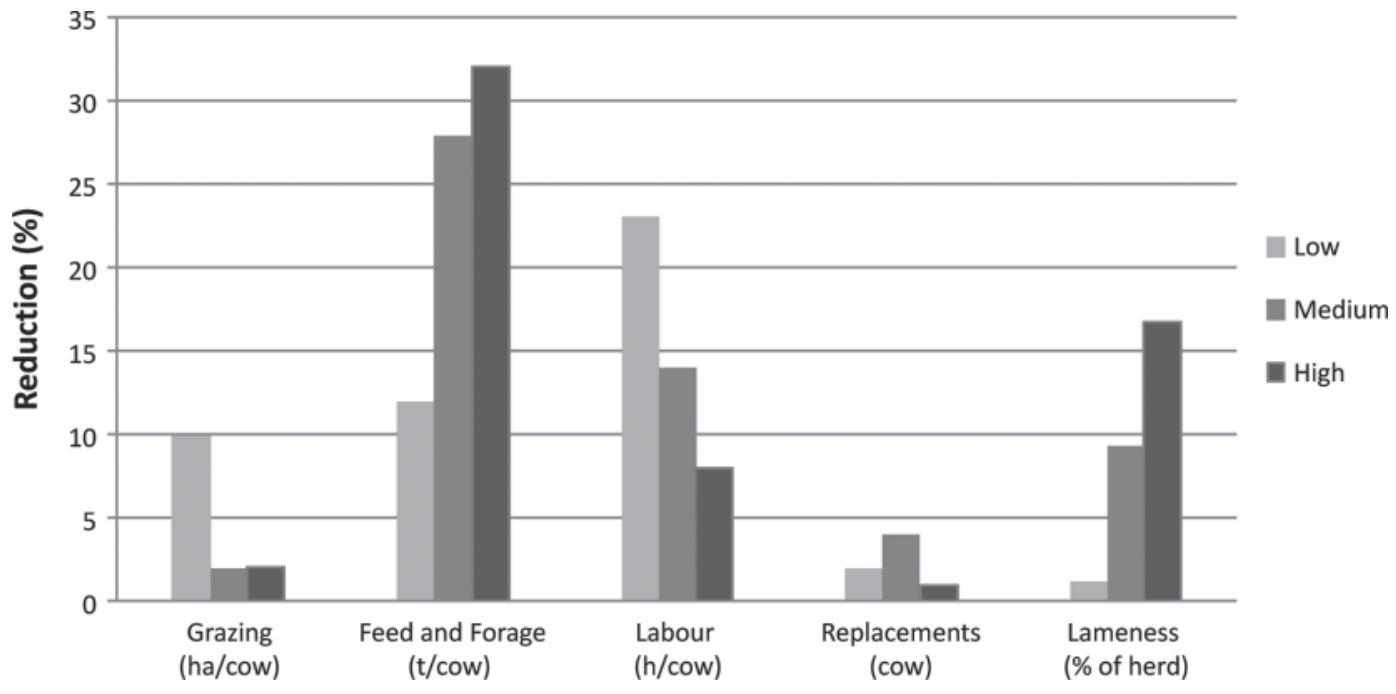

Figure 2. Mean level of input reduction (percentage reduction) required to become technically efficient by low, medium, and high lameness prevalence categories. 
signs of lameness or to implementing preventative measures such as hoof trimming or foot bathing. However, although seemingly inefficient from a partial input usage approach, a farmer who can reduce the percentage of severe lameness in the herd would improve efficiency at the whole enterprise level, simply due to less pressure on feeding inputs, the need for replacements, and improved milk yield.

\section{DISCUSSION}

Sustainable economic growth as a policy goal requires the development of indicators that can capture the synergies and trade-offs between economic, social, and environmental factors. Animal welfare is becoming more prominent on the agendas of consumers and policy makers and, as such, the effect of animal welfare status should be explored when developing measures of sustainable economic growth. This paper offers an approach that accounts for some of these relationships within one measure.

Animal welfare is usually composed of a suite of anthropogenically observed measures that act as proxy indicators of animal well-being. In this study, lameness, which is a highly visible and critical determinant of dairy cow welfare, has been used to understand the relationship between animal welfare and technical efficiency. Lameness is one of the most uncontroversial contributors to poor animal welfare within livestock management and, as such, could be actively managed to minimize occurrence. In the UK dairy industry, lameness levels are widely recognized as being too high (FAWC, 2009; Rutherford et al., 2009; Barker et al., 2010) However, the relationship between lameness and economic impact have found conflicting results (Warnick et al., 2001). With respect to dairy cattle in Britain, clear positive relationships exist between high animal welfare and high technical efficiency. This is complicated by the fact that certain inputs will be used less than efficiently to maintain these low levels of lameness. Some evidence suggests that farms with low proportions of lameness tend to have a higher labor to output ratio and a lower stocking density compared with those with higher levels of lameness. Indeed, high lameness farms have very little to gain from reducing either of these inputs to move toward the technical efficiency frontier. This is evidence that a whole-farm, rather than a partial indicator, approach to assessing the effect of lameness is required because the gain in yield still outweighs the comparative inefficiency of labor and grazing inputs.

The findings here are converse to those of Lawson et al. (2004), who measured the efficiency of Danish dairy farms. They found that dairy farms with higher levels of lameness and other factors tended to register higher levels of technical efficiency. They attributed this to farms with above average risk for these disorders as those with high milk production per cow or the loss of milk per day due to these disorders as too little to exert any significant effect on technical efficiency (Lawson et al., 2004). The major difference with this study is both the method of measurement and the treatment of lameness within the estimation of technical efficiency. Whereas Lawson et al. (2004) treat lameness as an explanatory variable, we have applied lameness as a controllable variable within the computation of technical efficiency and placed the minimization of lameness as a goal within farming, just as an efficient farm should minimize other inputs. (Some argument exists for treating lameness as an output of milk production. However, the aim would still be to minimize lameness while maximizing output even though an output-maximizing approach is not appropriate within present European Union agriculture, which aims for efficient use of resources and focuses on supply control measures.) Furthermore, Lawson et al. (2004) report mean technical efficiency results of $>0.9$ for the Danish dairy farming system. Strictly speaking, scores should not be compared across regions due to differences in technological possibilities (Battese et al., 2004; O'Donnell et al., 2008), but these higher mean scores can be related to the different structures within Danish dairying, the prevalence of national co-ops, and the higher intensity of production recorded on these farms rather than those within the British sample, which represents a range of input usage. However, it is clearly the case that, unlike in Lawson et al. (2004), the effect of lameness on the efficient conversion of inputs to outputs has a more significant impact on technical efficiency measurement.

\section{CONCLUSIONS}

A program that supports prescribed maximum levels of lameness will be beneficial to the farmer, cow welfare, and to society as a whole. The underlying requirement to reallocate labor time to prevent lameness within the herd will vary depending on farm characteristics and structures, but the farms observed here show such a significant improvement in resource use efficiency that extra investment may yield higher income flows for these farms. This may provide some evidence for supporting policies that encourage reduced lameness in cattle, such as lameness monitoring, or that aim to negate some of the cost from increasing labor time per cow needed in locomotion scoring of cattle. Support for labor training in locomotion scoring and welfare observance may encourage uptake of management methods that offer societal benefits. In addition, from a market perspec- 
tive, higher levels of animal welfare provide a basis for incurring a premium. This study finds that pursuing a lameness-monitoring strategy will have the benefit of more efficient resource use, and this will therefore have effects on the environmental and greenhouse gas requirements of dairy farming.

\section{ACKNOWLEDGMENTS}

We thank all the participating farmers and Lorna Sherwood, Lindsay Maggs, Mhairi Jack, and Jenny Gibbons from the Sustainable Livestock Systems Group, SAC Edinburgh for assisting with farm data collection. We gratefully acknowledge funding from Defra and the Scottish Government.

\section{REFERENCES}

Archer, S., N. Bell, and J. Huxley. 2010. Lameness in UK dairy cows: A review of the current status. In Pract. 32:492-504.

Banker, R. J., and R. C. Moray. 1986. The use of categorical variables in data envelopment analysis. Manage. Sci. 32:1613-1627.

Barker, Z. E., K. A. Leach, H. R. Whay, N. J. Bell, and D. C. J. Main. 2010. Assessment of lameness prevalence and associated risk factors in dairy herds in England and Wales. J. Dairy Sci. 93:932-941.

Barnes, A. P. 2008. Technical efficiency estimates for Scottish agriculture: A note. J. Agric. Econ. 59:370-376.

Barnes, A. P., D. M. Moran, and C. F. E. Topp. 2006. Does multifunctionality affect technical efficiency. J. Environ. Manage. 80:287-294.

Battese, G. E., D. S. P. Rao, and C. J. O'Donnell. 2004. A metafrontier production function for estimation of technical efficiencies and technology potentials for firms operating under different technologies. J. Prod. Anal. 21:91-103.

Bravo-Ureta, B. E., and L. Rieger. 1991. Dairy farm efficiency measurement using stochastic frontiers and neoclassical duality. Am. J. Agric. Econ. 73:421-428.

Charnes, A., W. W. Cooper, and E. Rhodes. 1978. Measuring the efficiency of decision making units. Eur. J. Oper. Res. 2:429-444.

Cloutier, L. M., and R. Rowley. 1993. Relative technical efficiency: Data envelopment analysis and Quebec's dairy farms. Can. J. Agric. Econ. 42:169-176.

Coelli, T. J., D. S. Prasada Rao, and G. E. Battese. 1998. An Introduction to Efficiency and Productivity Analysis. Kluwer Academic Publishers, London, UK.

Cooper, W. W., L. M. Seiford, and K. Tone. 2000. Data Envelopment Analysis. A Comprehensive Text with Models, Applications, References and DEA-Solver Software, Kluwer Academic Publishers, London, UK.

Cuesta, R. A. 2000. A production model with firm-specific temporal variation in technical inefficiency: With application to Spanish dairy farms. J. Prod. Anal. 13:139-158.

De Koeijer, T. J., G. A. A. Wossink, A. B. Smit, S. R. M. Janssens, J. A. Renkema, and P. C. Struik. 2003. Assessment of the quality of farmers' environmental management and its effects on resource use efficiency: A Dutch case study. Agric. Syst. 78:85-103.

Enting, H., D. Kooij, A. A. Dijkhuizen, R. B. M. Huirne, and E. N. Noordhuizen-Stassen. 1997. Economic losses due to clinical lameness in dairy cattle. Livest. Prod. Sci. 49:259-267.

Farrell, M. J. 1957. The measurement of productive efficiency. J. Royal Stat. Soc. A 120:253-281.

FAWC. 2009. Opinion on the Welfare of the Dairy Cow. Farm Animal Welfare Council, Millbank, London, UK.

Fourichon, C., H. Seegers, and X. Malher. 2000. Effects of disease on reproduction in the dairy cow: A meta-analysis. Theriogenology 53:1729-1759.
Fraser, I., and D. Cordina. 1999. An application of data envelopment analysis to irrigated dairy farms in Northern Victoria, Australia. Agric. Syst. 59:267-282.

Green, L. E., J. Borkert, G. Monti, and N. Tadich. 2010. Associations between lesion-specific lameness and the milk yield of 1,635 dairy cows from seven herds in the Xth region of Chile and implications for management of lame dairy cows worldwide. Anim. Welf. 19:419-427.

Green, L. E., V. J. Hedges, Y. H. Schukken, R. W. Blowey, and A J. Packington. 2002. The impact of clinical lameness on the milk yield of dairy cows. J. Dairy Sci. 85:2250-2256.

Hallam, D., and F. Machado. 1996. Efficiency analysis with panel data: A study of Portuguese dairy farms. Eur. Rev. Agric. Econ. 23:79-93.

Hansson, H. 2007. The links between management's critical success factors and farm level economic performance on dairy farms in Sweden. Acta Agric. Scand. C 4:77-88.

Hansson, H., and B. Ohlmer. 2008. The effect of operational managerial practices on economic, technical and allocative efficiency at Swedish dairy farms. Livest. Sci. 118:34-43.

Hansson, H., M. Szczensa-Rundberg, and C. Nielsen. 2010. Which preventive measures against mastitis can increase the technical efficiency of dairy farms? Animal 5:632-640.

Hernandez, J., J. K. Shearer, and D. W. Webb. 2002. Effect of lameness on milk yield in dairy cows. J. Am. Vet. Med. Assoc. 220:640-644.

Jaforullah. M., and J. Whiteman. 1999. Scale efficiency in the New Zealand dairy industry: A non-parametric approach. Aust. J. Agric. Res. Econ. 43:523-541.

Kuosmanen, T., and M. Kortelainen. 2011. Stochastic non-smooth envelopment of data: Semi-parametric frontier estimation subject to shape constraints. J. Prod. Analy.

Langford, F. M., K. M. D. Rutherford, M. C. Jack, L. Sherwood, A. B Lawrence, and M. J. Haskell. 2009. A comparison of management practices and winter housing on organic and non-organic dairy farms in the UK. J. Dairy Res. 76:6-14.

Lawson, L. G., J. F. Aggerb, M. Lunda, and T. Coelli. 2004. Lameness, metabolic and digestive disorders, and technical efficiency in Danish dairy herds: A stochastic frontier production function approach. Livest. Prod. Sci. 91:157-172.

Leach, K. A., H. R. Whay, C. M. Maggs, Z. E. Barker, E. S. Paul, A. K. Bell, and D. C. J. Main. 2010a. Working towards a reduction in cattle lameness: 1 . Understanding barriers to lameness control on dairy farms. Res. Vet. Sci. 89:311-317.

Leach, K. A., H. R. Whay, C. M. Maggs, Z. E. Barker, E. S. Paul, A. K. Bell, and D. C. J. Main. 2010b. Working towards a reduction in cattle lameness: 2. Understanding dairy farmers' motivations. Res. Vet. Sci. 89:318-323.

Lober, G., and M. Staat. 2010. Integrating categorical variables in data envelopment analysis models: A simple solution technique. Eur. J. Oper. Res. 202:810-818.

Manson, F. J., and J. D. Leaver. 1988. The influence of concentration amount and clinical lameness in dairy cattle. Anim. Prod. $47: 185-190$.

O'Donnell, C. J., D. S. P. Rao, and G. E. Battese. 2008. Metafrontier frameworks for the study of firm-level efficiencies and technology ratios. Empir. Econ. 34:231-255.

Piot-Lepetit, I., D. Vermersch, and R. D. Weaver. 1997. Agriculture's environmental externalities: DEA evidence for French agriculture. Appl. Econ. 29:331-338.

Rajala-Schultz, P. J., Y. T. Gröhn, and C. E. McCulloch. 1999. Effects of milk fever, ketosis, and lameness on milk yield in dairy cows. J. Dairy Sci. 82:288-294.

Reinhard, S., C. A. Knox Lovell, and G. J. Thijssen. 2000. Environmental efficiency with multiple environmentally detrimental variables: Estimated with SFA and DEA. Eur. J. Oper. Res. 121:287-303.

Rutherford, K. M. D., F. M. Langford, M. C. Jack, L. Sherwood, A B. Lawrence, and M. J. Haskell. 2008. Hock injury prevalence and associated risk factors on organic and nonorganic dairy farms in the United Kingdom. J. Dairy Sci. 91:2265-2274. 
Rutherford, K. M. D., F. M. Langford, M. C. Jack, L. Sherwood, A. B. Lawrence, and M. J. Haskell. 2009. Lameness prevalence and risk factors in organic and non-organic dairy herds in the United Kingdom. Vet. J. 180:95-105.

Simar, L., and P. W. Wilson. 1998. Sensitivity analysis of efficiency scores: How to bootstrap in nonparametric frontier models. Manage. Sci. 44:49-61.

Tauer, L. W., and J. J. Hanchar. 1995. Nonparametric technical efficiency with $\mathrm{K}$ firms, $\mathrm{N}$ inputs, and $\mathrm{M}$ outputs: A simulation. Agric. Resour. Econ. Rev. 24:185-189.
Warnick, L. D., D. Janssen, C. L. Guard, and Y. T. Gröhn. 2001. The effect of lameness on milk production in dairy cows. J. Dairy Sci. 84:1988-1997.

Whay, H. R., D. C. J. Main, L. E. Green, and A. J. F. Webster. 2003. Assessment of the welfare of dairy cattle using animal-based measurements: Direct observations and investigation of farm records. Vet. Rec. 153:197-202. 